\title{
Model Calculations for the \\ Explosive Generator-Driven \\ Dense Plasma Focus
}

\author{
John Brownell \\ Rolf Landshoff*
}

This work was performed in August 1978.

"Consultant. 525 East Crescent Drive, Palo Alto, CA 94301.

!ல囚

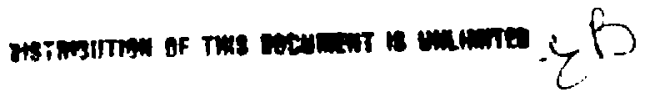




\title{
MODEL CALCULATIONS FOR THE \\ EXPLOSIVE GENERATOR-DRIVEN DENSE PLASMA FOCUS
}

by

John Brownell and Rolf Landshoff

\begin{abstract}
A model was developed to aid in the design and interpretation of explosive generator-driven dense plasma focus experiments. Several models were investigated, but the one presented here employs a plane sheath propogating along the barrel of a Mather-type gun, entraining a constant fraction of the swept-up gas and carrying the return current between the electrodes. The motion of the sheath is determined from the momentum equation using the integrated magnetic force on the sheath. The solutions are obtained both numerically and by an approximate analytic procedure, and the performance of the system has been determined as a function of the switching time when the generator is connected to the dense plasma focus.
\end{abstract}

The model presented in this report gives a zero-order approximation to the operating conditions of the explosive generator-driven dense plasma focus and has been used by Group M-6 at Los Alamos to establish a starting point for their experiments.

The arrangement under consideration consists of an explosive generator $G$, a ballast inductor $B$, and a Mather-type coaxial gun $F$, with an equivalent circuit as shown in Fig. 1. The inductance of $G$ is assumed to be

$$
\mathbf{L}_{\mathbf{G}}=\mathbf{L}_{\mathbf{G O}}\left(1-\frac{t}{t}\right)
$$

independent of the back pressure of the compiessed magnetic field. The initial inductance can be calculated from the expression

$$
L_{G O}=\mu_{n} / \eta\left[1-\frac{\eta}{\pi}\left(\ln \frac{1}{\eta}+1.5\right)\right] \text {, }
$$




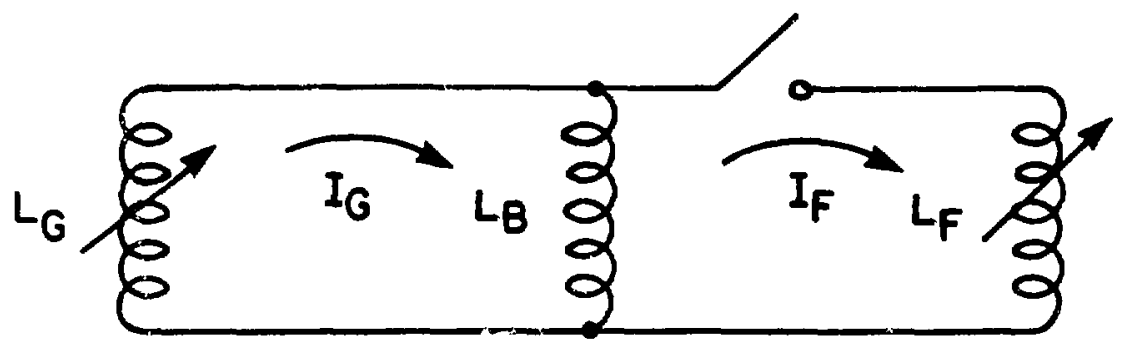

Fig. I. Equivalent circuit of gerterator, ballast, and Mather gun.

where $\eta=x_{0} / w, x_{0}$ is the initial distance between the generator plates, and $w$ and $l$ are the width and length of the plates. The length is the dimension in the direction of the current flow. The time $\tau$ depends on the strength of the explosive charges and construction details of the generator; it is typically 1-10 $\mu$ s.

At the time the switch is closed the generator current is

$$
I_{1}=I_{o}\left(\frac{L_{G o}+L_{\mathbf{b}}}{L_{G s}+L_{B}}\right) \text {, }
$$

where $I_{0}$ is the current at $t=0$ and $L_{G s}$ is the value of $L_{G}$ at $t=t_{s}$. The ballast inductance $L_{b}$ is assumed to be independent of time. After the dense plasma focus is connected, the return current between the two electrodes is assumed to be concentrated in a plane sheath propagating along the gun barrel where the sheath entrains a constant fraction $k$ of the swept-up gas. If the distance of the sheath from the breech of the gun is $\bar{z}$ and the radii of the outer and the central electrodes are $R_{0}$ and $R_{c}$, the inductance of $F$ is

$$
\mathbf{L}_{\mathrm{F}}=\mathbb{Z} \quad . \quad \mathrm{f}=\frac{\mu_{\mathrm{o}}}{2 \pi} \ln \left(\frac{\mathrm{R}_{\mathrm{o}}}{\mathrm{R}_{\mathrm{c}}}\right) .
$$

and the mass of the sheath is

$$
M_{F}=m Z . \quad m=\pi k p_{o}\left(R_{o}^{2}-R_{c}^{2}\right) .
$$

If we set $L_{G O}=\alpha \tau L_{1}$ and $f=\delta L_{-3}$, the cutrent in the gun as obtained from flux conservation is

$$
\mathrm{I}_{\mathrm{F}}=\mathrm{DI}_{1} \text {, }
$$

where

$$
D=\frac{a\left(t-t_{3}\right)}{a(\tau-t)+\delta Z[1+\alpha(\tau-t)]}
$$

"Equation (2) is an approximation to a more elaborate expression developed ty R. S. Caird, Los Alamos National Laboratory. 
and

$$
I_{1}=\frac{1+\alpha \tau}{1+\alpha\left(\tau-t_{s}\right)} I_{0} .
$$

The sheath motion is given by the momentum equation

$$
\frac{d}{d t}\left(M_{F} \dot{Z}\right)=F
$$

where

$$
F=\frac{f}{2} I_{F}^{2}
$$

is obtained by entering the field

$$
B=\mu_{0} \frac{I_{F}}{2 \pi R}
$$

into the relation

$$
F=\frac{1}{2 \mu_{0}} \int B^{2} 2 \pi R d R
$$

The momentum equation thus assumes the form

$$
\frac{\mathrm{d}}{\mathrm{dt}}(\mathrm{ZZ})=\frac{\mathrm{f}}{2 m} \mathrm{I}_{\mathrm{F}}^{2}
$$

After $Z$ has been determined (numerically or analytically as, for example, in the Appendix), various quantities of interest can be calculated, such as the energy deposited into $F$, the current $I_{F}$, and the rate of change of $L_{F}$.

The rate at which energy flows into $F$ is

$$
V_{F} I_{F}=I_{F} \frac{d}{d t}\left(L_{F} I_{F}\right)
$$

Integrating with respect to time, the energy in F can be expressed as the sum of two terms

$$
\int I_{F} \frac{d}{d t}\left(L_{F} I_{r}\right) d t:=E_{M}+W \text {, }
$$

where

$$
E_{M}=\frac{1}{2} L_{F} I_{F}^{2}
$$


is the stored magnetic energy, and

$$
W=\frac{1}{2} \int I_{F}^{2} \frac{d L_{F}}{d t} d t
$$

is the work done by the magnetic pressure, which produces both thermal and directed kinetic energy.

In addition. the quantities $I_{F}$ and $\dot{L}_{F}$ can be calculated from

$$
I_{\mathbf{F}}=I_{1} \sigma /\left\{1-\sigma+\delta z\left[1-\sigma+\frac{1}{a\left(t-t_{s}\right)}\right]\right\}
$$

with $I_{1}$ as given by $\mathrm{Eq}(8)$, and

$$
L_{F}=f \sqrt{\frac{f}{3 m}} \frac{\left(1+\frac{3}{2} \sigma+\frac{3}{2} \xi \sigma^{2}\right)}{\sqrt{1+1.2 \sigma+\varepsilon \sigma^{2}}},
$$

where $\sigma$ and $\xi$ are defined in the Appendix. These quantities are of particular importance when the generator plates make contact and the voltage drops to zero, at which time no more power is delivered to the load F. At the time $t_{c}$ at which this contact occurs, the inductance $L_{G}$ still has a finite value

$$
L_{G i C}=\mu_{o} \frac{x_{c}}{w}
$$

The residual $x_{c}$ in this expression is made up of two parts, the thickness $x_{1}$ of a sheet of insulating material placed between the two plates and the skin depth $x_{3}$ by which the flux has diffused into the generator plates (twice the skin depth per plate). Typically, $x_{i}$ is $10^{-3} \mathrm{~m}$, and for aluminum.

$$
x_{s} \approx 1.4 \times 10^{-4} \sqrt{\frac{I_{0} \tau}{w}} m,
$$

where all quantities are given in mks units."

The residual inductance can be accounted for by terminating the calculation at

$$
t_{c}=\tau\left(1-\frac{L_{G c}}{L_{G O}}\right)
$$

or

$$
\sigma_{c}=\left(I-\frac{L_{G C}}{L_{G O}}-\frac{t_{s}}{\tau}\right) / 1-\frac{t_{s}}{\tau} .
$$

-This form is due to an investigation by C. M. Fowler, Los Alamos National Laboratory. 
The model developer above was applied to a setup in which the dimensions and the coilapse time of the generator and the dimensions of the Mather gun remain fixed. The actual values as suggested by Group M-6 are

Generator: $t=0.528 \mathrm{~m}, w=0.1524 \mathrm{~m}, \mathrm{x}_{\mathrm{o}}=0.0762 \mathrm{~m}$

$$
x_{c}=0.002 \mathrm{~m}, \tau=10^{-3} \mathrm{~s}
$$

Mather Gun: $R_{0}=0.0762 \mathrm{~m}, R_{c}=0.0508 \mathrm{~m}, \mathrm{~d}=0.3 \mathrm{~m}$.

The last quantity is the length of the center electrode; we will describe later how this is to be used. The switch time $t_{s}$, the ballast inductance $L_{B}$, and the initial gas density $\rho_{0}$ in the gun are considered as tuning parameters. The entrainment factor, alsc called the plowing efficiency, was arbitrarily taken to be $k=1$ because the results depend only on the product $k \rho_{0}$. Actually, the value of $k$ is not known and may depend on time, but in the present model, the possibility is not considered. In principle, the initial generator current $I_{o}$ could be used for tuning, but a look at the equations shows that the performance depends on the ratio $I_{0}{ }^{2} / \rho_{0} k$ and there is no need for changing both $\rho_{0}$ and $I_{0}$. The current, therefore, is considered as fixed at a value $\mathrm{l}_{\mathrm{o}}=7.5 \times 10^{5} \mathrm{~A}$.

We have assumed that the best results could be obtained if the plasma sheath reaches the end of the center electrode at the same time the power flow into the gun ends; that is, if $Z\left(t_{c}\right)=d$. This relation has been used to determine $t_{s}$ for given values of $\rho_{o}$ and $L_{B}$. The set of curves in Fig. 2 shows the results for different values of $\rho_{0}$ plotted against $L_{B}$. The curves are labeled by the gas pressure of room-temperature deuterium expressed in torr. The ratio was taken to be $p / \rho_{0}=(3 / 7) \times 10^{4} \mathrm{torr} /\left(\mathrm{kg} / \mathrm{m}^{3}\right)$. In Figs. 2. 3, and

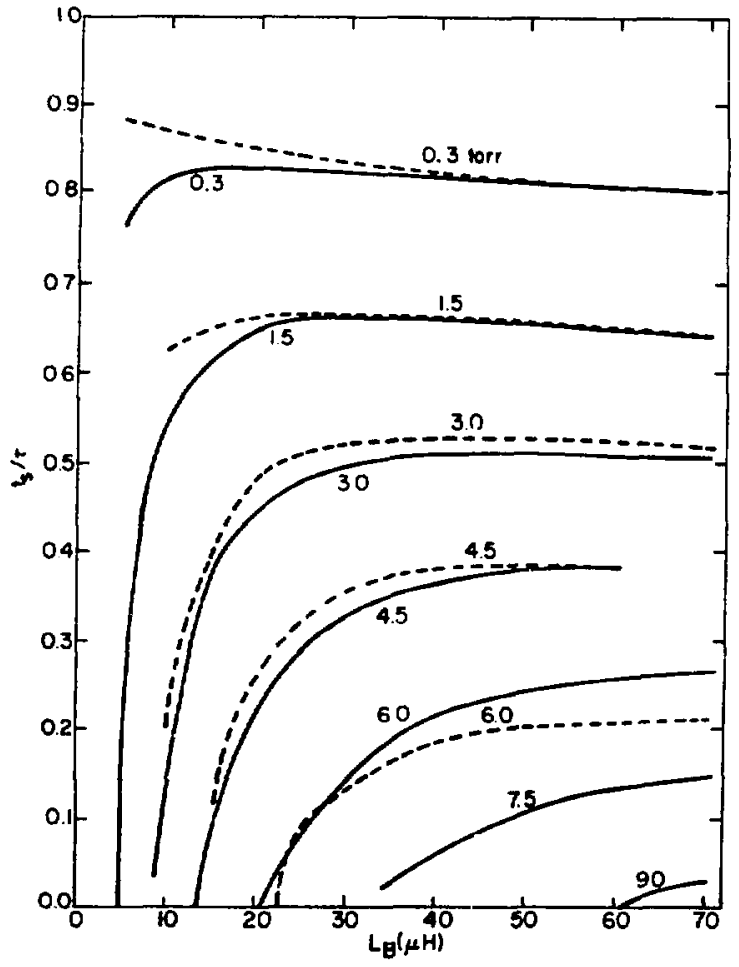

Fig. 2. Switch time (dashed lines represent analytic approximation, solid lines are numerical integration).

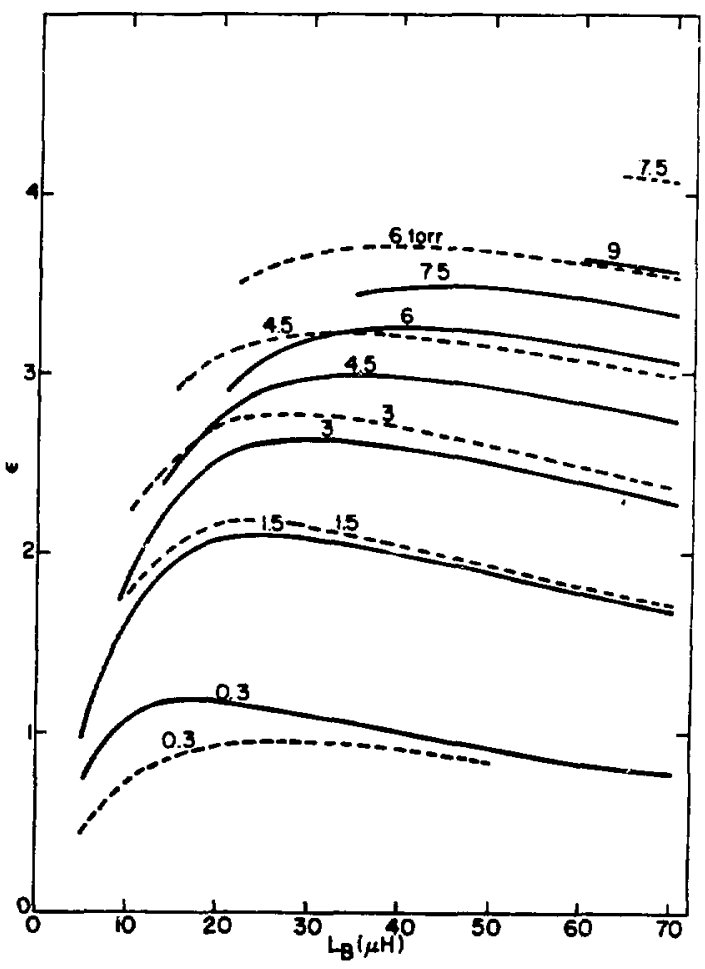

Fis. 3. Energy-gain factor (dashed lines represent analyric approximation, solid lines are numerical integration). 


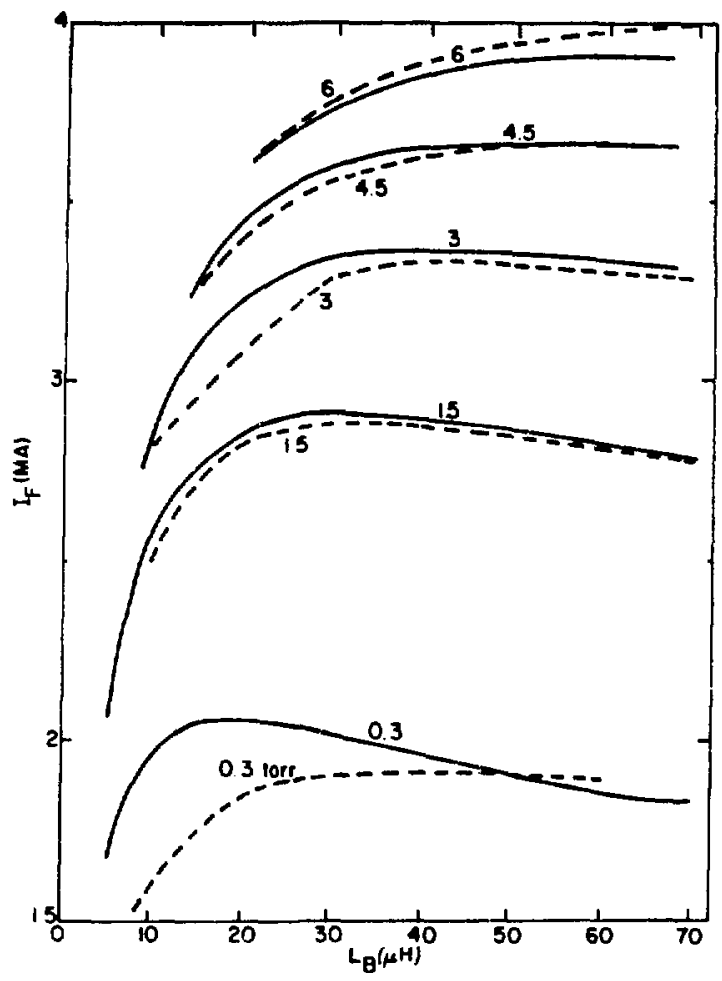

Fig. 4. Peak current (dashed lines represent analytic approximation. solid lines are numerical inegration).

4, the analytic approximations are given as dashed lines and the results of the numerical integrations are given as solid lines. As show $\pi$, the analytic approximation agrees quite well. The purpose of the dense plasma focus arrangement is to produce a maximum fusion yield at the focus. At the end of the first stage (the rundown). it is not possible to predict precisely how to achieve this. It appears likely, however, that one should optimize the ratio of the energy transfer to the gun to the initially available electromagnetic energy

$$
E_{0}=\frac{1}{2}\left(L_{G o}+L_{0}\right) J_{0}^{2}
$$

We therefore define an energy-gain factor

$$
\varepsilon=\frac{E_{M}+W}{E_{o}}
$$

Figure 3 shows graphs of this factor for the switch times shown in Fig. 2, and peak currents are shown in Fig. 4. The curves show that the peak values of $\varepsilon$ rise with $p$, but one is limited by the fact that $t_{3}$ has to be positive. Thus, for the chosen dimensions, it appears impractical to go beyond 6 torr, where one reaches a gain factor of about 3.7. In an arrangement where the electromagnetic energy is fed directly from a capacitor to the gun, the fraction of the energy fed to the gun has been estimated ${ }^{*}$ to be at most $\varepsilon$ $=0.586$; that is, the explosion generator setup is about six times as effective.

-This information provided by R. H. Gerwin, Los Alamos National Laboratory. 
The energy gain is derived from the work done by the explosive and the efficiency in terms of that energy is quite small. However, that is not an important consideration here because the explosive energy is readily available.

When comparing the predictions of the model, consider that the quantity designated as the pressure is really the product of the true pressure and the entrainment factor. The effective value of this factor can be obtained by observing the sheath distance $Z$ at generator burn-out time for a set combination of $t_{s}, L_{B}$. and $p$ and dividing the $k p$ for which one expects the observed $Z$ by the true value of $p$. In Fig. 5, we have plotted curves of $Z$ vs $k p$ for $L_{n}=30 \mathrm{nH}$ and three values of $t_{s} / \tau$ that can be used for such a determination.

Note that the relation between $p$ and $\rho_{0}$ applies only to deuterium. For other gasses, one should represent the results in terms of density rather than pressure.

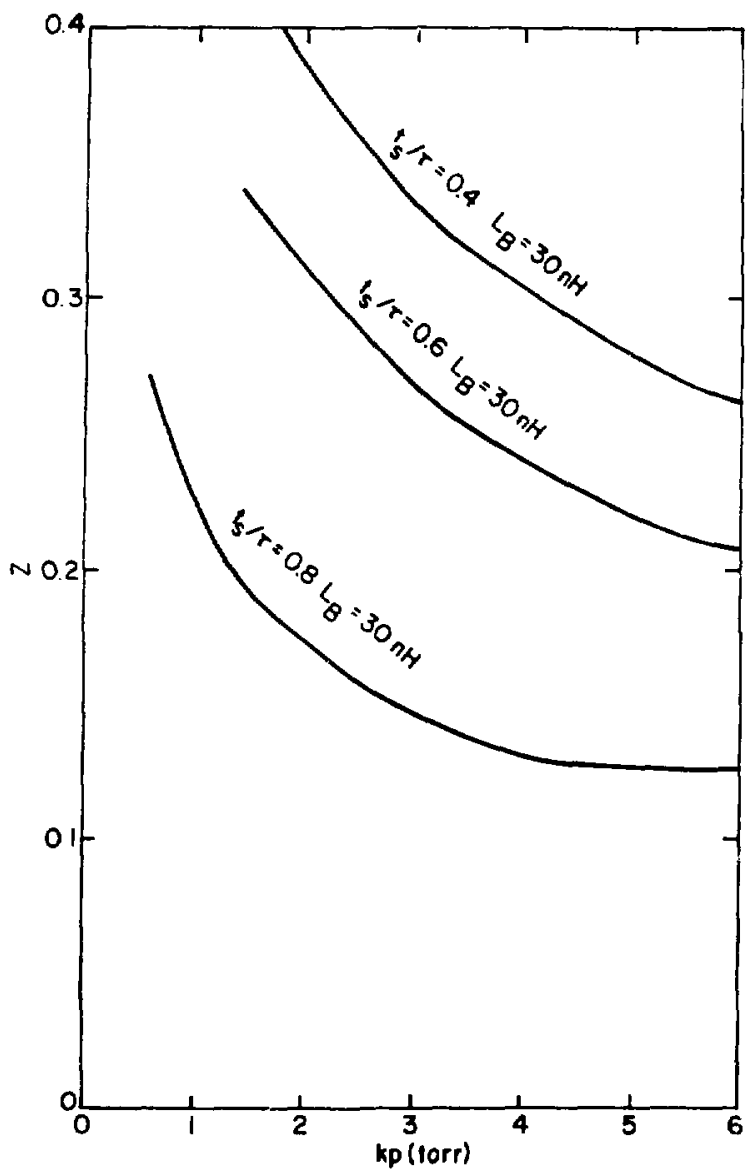

Fig. 5. Sheath distance at burn-out time vs kp. 


\section{APPENDIX}

\section{APPROXIMATE ANALYTIC SOLUTION}

We attempt to find an approximate solution to Eq. (13) by introducing the variable

$$
\sigma=\frac{t-t_{5}}{\tau-t_{5}}
$$

and by setting

$$
Z^{2}=\left(\frac{f}{12 m}\right) I_{1}^{2}\left(\tau-t_{s}\right)^{2} \sigma^{4}\left(1+1.2 \sigma+\xi \sigma^{2}\right)
$$

so that

$$
\frac{\mathrm{d}}{\mathrm{dt}}(\mathrm{ZZ})=\frac{\mathrm{d}^{2}}{\mathrm{dt}^{2}}\left(\frac{\mathrm{Z}^{2}}{2}\right)=\left(\frac{\mathrm{f}}{2 m}\right) \mathrm{I}_{1}^{2} \sigma^{2}\left(1+2 \sigma+\frac{5}{\mathrm{Z}} \xi \sigma^{2}\right) .
$$

By substituting Z from Eq. (A.2) with D [see Eq. (6)], we obtain the relation

$$
\begin{aligned}
& 1+2 \sigma+\frac{5}{2} \xi \sigma^{2}= \\
& \left\{1-\sigma+q_{0} \sigma^{2} \sqrt{1+1.2 \sigma+\xi \sigma^{2}}\left[1+\alpha\left(\tau-t_{3}\right)(1-\sigma)\right]\right\}^{-2}
\end{aligned}
$$

with

$$
\mathrm{q}_{\mathrm{o}}=\frac{\sigma}{\alpha} \sqrt{\frac{\mathrm{f}}{12 m}} \mathrm{I}_{1}
$$

which is satisfied to terms in first order in $\sigma$. The value of $\xi$ for which Eq. (A.4) is satisfied is not constant, which it would have to be if Eq. (A.2) were an exact solution. After comparing the approximate $Z$ with one obtained by a numerical solution of Eq. (13), we decided to determine $\xi$ by solving Eq. (A.4) for $\sigma=$ 0.8 ; that is, by solving

$$
2.6+1.6 \xi=\left(0.2+q_{1} \sqrt{1.96+0.64 \xi}\right)^{-2},
$$

where

$$
\mathrm{q}_{1}=0.64 \mathrm{q}_{0}\left[1+0.2 \alpha\left(\tau-\mathrm{t}_{\mathrm{s}}\right)\right]
$$


For large values of $q_{1}$, this procedure of obtaining $\xi$ must be modified because it would lead to negative values of $\left(\mathrm{d}^{2} / \mathrm{dt}^{2}\right)\left(\mathrm{Z}^{2}\right)$, which are physically impossible. We therefore change the relation so that in the limit $q_{1} \rightarrow \infty, \xi$ approaches -1.2 , which is the value for which the factor in Eq. (A.3) becomes zero when $\sigma=1$. A relation that does this and fits Eq. (A.6) for $q_{1}<0.4$ is

$$
\xi=\left(0.2+0.25 q_{1}+6.25 q_{1}^{2}\right)^{-1}-1.2
$$

